\title{
Protective Effect of Carvacrol on Renal Functional and Histopathological Changes in Gentamicin-Induced-Nephrotoxicity in Rats
}

\author{
Hassan Ahmadvand,,${ }^{1,2,}$ Majid Tavafi, ${ }^{3}$ Vahideh Asadollahi, ${ }^{4}$ Leila Jafaripour, ${ }^{5}$ Forouzan \\ Hadipour-Moradi, ${ }^{1}$ Reza Mohammadrezaei-Khoramabadi, ${ }^{6}$ Peyman Khosravi, ${ }^{6}$ Hadis Salehi, ${ }^{6}$ and \\ Ahmad Cheraghi ${ }^{1}$
}

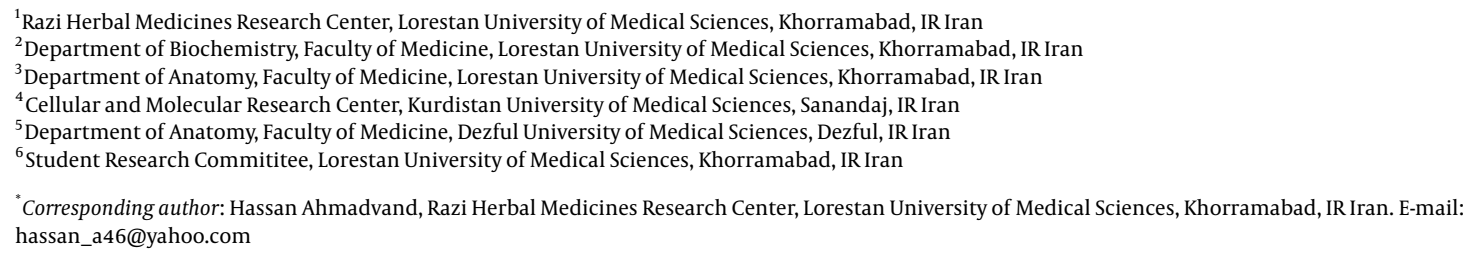

Received 2015 January 28; Accepted 2015 August 19.

\begin{abstract}
Background: Nephrotoxicity is one of the most important side effects of the use of gentamicin sulphate (GS) resulted in reactive oxygen species generation. Antioxidant compounds played effective roles in reduction of renal injuries caused by using of gentamicin. Carvacrol is a strong antioxidant compound.

Objectives: The aim of this study is to explore the effect of carvacrol inhibition in lesions of gentamicin-induced nephrotoxicity. Materials and Methods: In this experimental study, 32 male mature Sprague-Dawley rats were divided into 4 groups of 8; group1: control, group 2 sham received daily carvacrol injection $(74 \mathrm{mg} / \mathrm{kg}$ ) for 12 days, group 3 received daily GS injection (100 $\mathrm{mg} / \mathrm{kg}$ ) for 12 days, group 4 received daily GS (100 mg/kg) and carvacrol $(74 \mathrm{mg} / \mathrm{kg})$ for 12 days. After 12 days, rats were anaesthetized, blood sample were obtained and kidneys were removed then stained with hematoxylin and eosin method and then were studied histophatologically. Serum creatinine and urea were measured.

Results: Flow treatment of nephrotoxic animals with carvacrol could significantly inhibit leukocyte infiltration (9.42\%) and tubular necrosis (38.18\%) in comparison with the nephrotoxic untreated group. Carvacrol significantly decreased the levels of urea and creatinine in treated group compared with the nephrotoxic untreated group.

Conclusions: The findings showed that carvacrol alleviates loss of leukocyte infiltration (9.42\%) and tubular necrosis and exerts beneficial effects on kidney function test in nephrotoxic group.
\end{abstract}

Keywords: Carvacrol, Gentamicin, Nephrotoxicity, Rat

\section{Background}

Gentamicin sulphate (GS) is considered as one of the aminoglycoside antibiotics used to treat infection especially against Gram-negative bacteria such as Pseudomonas, Proteus and Serratia [1-3]. Nephrotoxicity is one of the most important side effects of the use of gentamicin resulted in reactive oxygen species generation such as superoxide anion, hydrogen peroxide and hydroxyl radicals in the kidney [4-9]. Gentamicin-induced nephrotoxicity increased the activity of superoxide dismutase [5, $10,11]$, glutathione peroxidase [12] and catalase in animal models. Thus; they are more sensitive to reactive oxygen species (ROS) effects. Gentamicin-induced nephrotoxicity increases the level of serum creatinine and urea, tubular necrosis and glomerular congestion but decrease glomerular filtration rate [13-15]. In studies done on antioxidant compounds such as curcumin [16] olive leaf extract [5], green tea and coenzyme Q10 [6] and rosmarinic acid [17] such as antioxidant compounds played effective roles in reduction or decrease of renal injuries caused by using of gentamicin.

A number of natural antioxidant such as vitamin E and phenolic compounds are known to have protective effects on liver injury and nephrotoxicity [18]. Chemical drugs as antioxidant have many side effects; therefore, screening for new natural antioxidants is still attractive because they are safe and good alternative for prevention of nephrotoxicity induced by GS. A growing body of research indicates that nutritional deficiencies such as antioxidants contribute to the development of nephrotoxicity.

Carvacrol or cymophenol (2-methyl-5-isopropyl phe- 
nol) is a predominant monoterpenic phenol which occurs in many essential oil of the family Labiatae including Origanum satureja, Thymbra, Thymus and Corydothymus species $[19,20]$. Carvacrol knows as a strong antioxidant has an antioxidant characteristic that its characteristic is similar to vitamin E, C and butyl hydroxyl toluene(BHT) [21, 22].

It seems that carvacrol also has such characteristic because most of the antioxidant has anti carcinogenic and antiplatelet characteristics [23,24]. The possible protective effects of carvacrol on structural lesions of kidney tissue in lesions of gentamicin-induced nephrotoxicity male rats have not yet been reported.

\section{Objectives}

Hence in this research, the inhibitory effect of carvacrol on structural lesions of kidney tissue caused by gentamicin-induced nephrotoxicity will be considered in an animal model.

\section{Materials and Methods}

In this experimental study, 32 male Sprague-Dawley rats $(200 \mathrm{~g}$ to $220 \mathrm{~g}$ ) were prepared from Pasteur Institute of Tehran and they were allowed to adapt themselves with the new location for 1 week. They were kept at a room temperature of $22^{\circ} \mathrm{C}$ and a humidity of $50 \pm 10 \%$ with 12-hours light/dark cycles. This study was approved by the animal ethics committee of Lorestan University of Medical Sciences and was in accordance with the national health and medical research council guidelines. The animals were randomly assigned to 4 groups (8 per group): 1- Control group 2-Sham group treated with $74 \mathrm{mg} / \mathrm{kg}$ carvacrol (purchased from Sigma Chemical Company) i.p. for 12 days without treatment with gentamicin. 3-Gentamicininduced nephrotoxicity received $100 \mathrm{mg} / \mathrm{kg}$ gentamicin i.p. for 12 days. 4- Nephrotoxicity group received $100 \mathrm{mg} / \mathrm{kg}$ gentamicin and $74 \mathrm{mg} / \mathrm{kg}$ carvacrol i.p. for 12 days. After 12 days treatment animals were anesthetized and blood samples of their hearts were obtained and keeps at room temperature for 30 minutes and then centrifuged at 3000 rpm for 15 minutes for serum separation. Their right kidneys were excised for tissue processing and were fixed in $10 \%$ formaldehyde solution after tissue processing of their kidney paraffin sections ( $5 \mu$ m thicknesses) were prepared and stained by hematoxylin and eosin.

\subsection{Renal Function Parameters}

Serum creatinine and urea were measured by autoanalyzer (Biotechnical instruments BT 1000) Parsazmoon, Tehran, Iran.

\subsection{Renal Histological Analysis}

After tissue processing of rat kidneys paraffin sections ( $5 \mu \mathrm{m}$ thicknesses) were prepared and stained by hematoxylin and eosin. Forty regions of proximal convoluted tubules near of each kidney at the magnification of $\times 400$ were observed to consider the histopathological changes such as eosinophilic casts, leukocyte infiltration and tubular necrosis. Caramel method was utilized to assess these changes semiquantitively [25]:

$0=$ no damage; 1 = damage mild, patchy isolated; $2=$ damage less than $25 \% ; 3=$ damage between $25 \%$ - 50\%; $4=$ more than $50 \%$ damage.

The results of each change were reported separately.

\subsection{Statistical Analysis}

All values are expressed as mean \pm SD. Data between groups were first tested Kruskal-Wallis one-way and then between two groups were analyzed by Mann-Whitney U test. Statistical analyses were performed using SPSS-16. A P value $<0.05$ was considered statistically significant.

\section{Results}

The level of serum urea is shown in Table 1. Our study demonstrated that the level of serum urea in untreated nephrotoxic rats was significantly (4.00-fold) higher than that of the control animals. Treatment of nephrotoxic animals with carvacrol could not significantly (57.10\%) inhibit serum urea in comparison with untreated nephrotoxic group. The level of serum urea in sham, similar to the level found in the nephrotoxic treated animals. The level of serum creatinine is shown in Table 1. Our study demonstrated that the level of serum creatinine in untreated nephrotoxic rats was significantly (4.44-fold) higher than that of the control animals. Treatment of nephrotoxic animals with carvacrol could not significantly inhibit serum creatinine in comparison with untreated nephrotoxic group.

The level of eosinophilic casts is shown in table 2. Our study demonstrated that the level of the eosinophilic casts in untreated nephrotoxic rats was significantly (72.16-fold) higher than that of the control animals. Treatment of nephrotoxic animals with carvacrol could not significantly inhibit eosinophilic casts in comparison with untreated nephrotoxic group. The level of eosinophilic casts in sham, similar to the level found in the nephrotoxic treated and untreated animals. The level of leukocyte infiltration is shown in Table 2. Our study demonstrated that the level of the leukocyte infiltration in untreated nephrotoxic rats 
Table 1. Effect of Carvacrol on Serum Urea and Creatinine During Gentamicin-Induced Nephrotoxicity ${ }^{\mathrm{a}}$

\begin{tabular}{lcc}
\hline Groups & Serum Urea, mg/dL & Serum Creatinin, mg/dL \\
\hline Control & $43.83 \pm 4.71^{\mathrm{b}}$ & $50.00 \pm 4.02^{\mathrm{b}}$ \\
\hline Sham & $175.43 \pm 39.47^{\mathrm{c}}$ & $0.61 \pm 0.07^{\mathrm{b}}$ \\
\hline Gentamicin-induced nephrotoxicity & $75.25 \pm 20.33^{\mathrm{b}, \mathrm{c}}$ & \\
\hline Gentamicin-induced nephrotoxicity treated & & \\
\hline $\begin{array}{l}{ }^{\mathrm{a}} \text { Values are expressed as Mean } \pm \text { SEM. } \\
{ }^{\mathrm{b}} \mathrm{P}<0.05 \text { as compared with gentamicin-induced nephrotoxicity without treatment group. } \\
{ }^{\mathrm{c}} \mathrm{P}<0.05 \text { as compared with control group. }\end{array}$ & \\
\hline
\end{tabular}

was significantly (23.87-fold) higher than that of the control animals. Treatment of nephrotoxic animals with carvacrol could not significantly inhibit leukocyte infiltration in comparison with untreated nephrotoxic group.

The level of leukocyte infiltration in sham, similar to the level found in the nephrotoxic treated and untreated animals. The level of tubular necrosis is shown in Table 2. Our study demonstrated that the level of the tubular necrosis in untreated nephrotoxic rats was significantly (12.82fold) higher than that of the control animals. Treatment of nephrotoxic animals with carvacrol could significantly (27.63\%) inhibit tubular necrosis is in comparison with untreated nephrotoxic group. The level of tubular necrosis in sham, similar to the level found in the nephrotoxic treated animals.

\section{Discussion}

According to the findings of this study, it can be concluded that GS injection in rats for 12 days can lead to renal disorder in rats. The con-current administration of GS with carvacrol $(74 \mathrm{mg} / \mathrm{kg}$ ) for 12 days at least partially resulted in decreased of renal injuries caused by GS therapy. In this study the level of both serum urea and tubular necrosis significantly decreased in gentamicin-inducednephrotoxicity groups treated with carvacrol compared to gentamicin-induced-nephrotoxicity groups none treated with carvacrol.

Although GS has an effective role in treatment of Gram negative bacteria, its administration has been limited because of increasing the chance of acute renal failure in 10\% - $20 \%$ of patients treated with this drug [26]. Gentamicininduced-nephrotoxicity can cause renal injuries due to ROS generation $[4,14,15]$. Antioxidant compounds such as melatonin [27], vitamin E and probacol [28] etc. have been utilized to decrease the rat of renal injuries caused by gentamicin-induced-nephrotoxicity.

Previous our study showed that Satureja khozestanica essential oil has hypolipidemic effects and reduce glomerular hypertrophy, glomerulosclerosis and loss of glomerular number in untreated diabetic nephropathy rats $[29,30]$. Also, previous our study showed that $S$. khozestanica essential oil is a source of potent antioxidants and prevents the oxidation of LDL in vitro [31]. The main component of S. khozestanica essential oil is carvacrol as a good antioxidant [32]. Although carvacrol knows as a strong antioxidant $[21,22]$, it seems that it can decrease renal injuries caused by gentamicin-induced-nephrotoxicity due to its antioxidant characteristic. In current study, the level of serum urea and creatinine significantly increased in gentamicin-induced nephrotoxicity group that such findings were similar to the previous studies that the increase in level of serum urea and creatinine maybe not only due to the glomerular injuries, but also due to the decrease of glomerular filtration $[5,16,17,33$, 34]. Gentamicin-induced nephrotoxicity treated with carvacrol group the level of urea significantly declined. It can be speculated that the decrease of serum urea likely may be due to antioxidant characteristic of carvacrol because in other studies antioxidants such as garlic extract [16], olive leaf extract [5], rosmaric acid [17], and aminoguanidin [35] also have reported a decline level of serum urea and creatinine. In this study, the level of eosinophilic casts, leukocyte infiltration and tubular necrosis significantly increased in nephrotoxic group, while carvacrol therapy only significantly decreased the level of tubular necrosis. In similar studies that considered the effects of different antioxidants, the decrease in the rate of tissue injuries caused by gentamicin-induced-nephrotoxicity by using antioxidants have also been reported $[6,16,17,28,36]$.

From these studies it can be concluded that the decrease in the level of tubular necrosis after treatment with carvacrol may be due to its antioxidant characteristic from the current study. We can conclude that administration of carvacrol and GS in rats can prevent gentamicin-induced renal toxin at least partially.

Antioxidant therapy is one of the most important treatment strategies for kidney damage patients for the pre- 
Table 2. Effect of Carvacrol on Histopathological Parameters During Gentamicin-Induced Nephrotoxicity ${ }^{\mathrm{a}}$

\begin{tabular}{|c|c|c|c|}
\hline Groups & Eosinophilic Casts & Leukocyte Infiltration & Tubular Necrosis \\
\hline Control & $0.042 \pm 0.041^{\mathrm{b}}$ & $0.125 \pm 0.048^{\mathrm{b}}$ & $0.188 \pm 0.07^{\mathrm{b}}$ \\
\hline Sham & $3.512 \pm 0.0893^{c}$ & $2.272 \pm 0.109^{b, c}$ & $2.483 \pm 0.122^{\mathrm{b}, \mathrm{c}}$ \\
\hline Gentamicin-induced nephrotoxicity & $3.031 \pm 0.094$ & $2.984 \pm 0.106$ & $3.333 \pm 0.094$ \\
\hline Gentamicin-induced nephrotoxicity treated & $3.125 \pm 0.147^{c}$ & $2.727 \pm 0.133^{\mathrm{c}}$ & $2.412 \pm 0.153^{\mathrm{b}, \mathrm{c}}$ \\
\hline
\end{tabular}

vention and slowing of kidney damage complications progression. However, the possible underlying molecular protective mechanisms of carvacrol were not completely explained by our results. Also our results showed that carvacrol has some beneficial effects in prevention and slowing of kidney damage complications progression but it can not be introduced to nephrotoxic patients for inhibition and progression of kidney damage complications.

This study showed that carvacrol has beneficial effects, in decreasing the elevated serum urea and tubular necrosis but has not beneficial effects, in decreasing the elevated serum creatinine, eosinophilic casts, and leukocyte infiltration in nephrotoxic rats. Hence, this study showed that carvacrol can probably decrease some nephrotoxic complication such as nephropathy in nephrotoxic patients.

\section{Acknowledgments}

This study approved with grant number 91/6 in the Lorestan University of Medical Sciences. Special thanks to Lorestan University of Medical Sciences for the financial support, khorramabad, Iran. The authors thank the head and staff of Razi Herbal medicines research center of Lorestan Medical University.

\section{Footnotes}

Authors' Contribution Hassan Ahmadvand, Majid Tavafi and Leila Jafaripour provided conception and design of the study and revised the article, Vahideh Asadollahi, Forouzan Hadipour-Moradi and Reza MohammadrezaeiKhoramabadi participated in data collection and drafting the manuscript, also the data analysis and interpretation performed by Peyman Khosravi, Hadis Salehi and Ahmad Cheraghi.

Funding/Support This paper had been done by personal expenses.

\section{References}

1. Tangden T, Enblad P, Ullberg M, Sjolin J. Neurosurgical gram-negative bacillary ventriculitis and meningitis: a retrospective study evaluating the efficacy of intraventricular gentamicin therapy in 31 consecutive cases. Clin Infect Dis. 2011;52(11):1310-6. doi: 10.1093/cid/cir197. [PubMed: 21540208].

2. Corona PS, Espinal L, Rodriguez-Pardo D, Pigrau C, Larrosa N, Flores X Antibiotic susceptibility in gram-positive chronic joint arthroplasty infections: increased aminoglycoside resistance rate in patients with prior aminoglycoside-impregnated cement spacer use. I Arthroplasty. 2014;29(8):1617-21. doi: 10.1016/j.arth.2014.03.029. [PubMed: 24798194].

3. Shrestha B, Haylor J. Experimental rat models of chronic allograft nephropathy: a review. Int J Nephrol Renovasc Dis. 2014;7:315-22. doi: 10.2147/IJNRD.S65604. [PubMed: 25092995].

4. Tavafi M. Protection of renal tubules against gentamicin induced nephrotoxicity. J Renal Inj Prev. 2013;2(1):5-6. doi:10.12861/jrip.2013.03. [PubMed: 25340112].

5. Tavafi M, Ahmadvand H, Toolabi P. Inhibitory effect of olive leaf extract on gentamicin-induced nephrotoxicity in rats. Iran J Kidney Dis 2012;6(1):25-32. [PubMed: 22218116].

6. Ahmadvand H, Ghasemi Dehnoo M, Dehghani A, Bagheri S, Cheraghi RA. Serum paraoxonase 1 status and its association with atherogenic indexes in gentamicin-induced nephrotoxicity in rats treated with coenzyme Q10. Ren Fail. 2014;36(3):413-8. doi: 10.3109/0886022X.2013.865154. [PubMed: 24320085].

7. Stojiljkovic N, Stoiljkovic M, Randjelovic P, Veljkovic S, Mihailovic D. Cytoprotective effect of vitamin $C$ against gentamicin-induced acute kidney injury in rats. Exp Toxicol Pathol. 2012;64(1-2):69-74. doi: 10.1016/j.etp.2010.06.008. [PubMed: 20619622].

8. Moreira MA, Nascimento MA, Bozzo TA, Cintra A, da Silva SM, Dalboni MA, et al. Ascorbic acid reduces gentamicin-induced nephrotoxicity in rats through the control of reactive oxygen species. Clin Nutr. 2014;33(2):296-301. doi: 10.1016/j.clnu.2013.05.005. [PubMed: 23810398].

9. Salem EA, Salem NA, Kamel M, Maarouf AM, Bissada NK, Hellstrom WJ, et al. Amelioration of gentamicin nephrotoxicity by green tea extract in uninephrectomized rats as a model of progressive renal failure. Ren Fail. 2010;32(10):1210-5. doi: 10.3109/0886022X.2010.517350. [PubMed: 20954983].

10. Aygun FO, Akcam FZ, Kaya O, Ceyhan BM, Sutcu R. Caffeic acid phenethyl ester modulates gentamicin-induced oxidative nephrotoxicity in kidney of rats. Biol Trace Elem Res. 2012;145(2):211-6. doi: 10.1007/s12011-011-9172-0. [PubMed: 21870154].

11. Ojano-Dirain CP, Antonelli PJ, Le Prell CG. Mitochondria-targeted antioxidant MitoQ reduces gentamicin-induced ototoxicity. Otol Neurotol. 2014;35(3):533-9. doi: 10.1097/MAO.0000000000000192. [PubMed: 24518411]. 
12. Gheissari A, Mehrasa P, Merrikhi A, Madihi Y. Acute kidney injury: A pediatric experience over 10 years at a tertiary care center. J Nephropathol. 2012;1(2):101-8. doi: 10.5812/nephropathol.7534. [PubMed: 24475397].

13. Nasri H. Acute kidney injury and beyond. J Ren Inj Prev. 2012;1(1):1-2.

14. Romero F, Perez M, Chavez M, Parra G, Durante P. Effect of uric acid on gentamicin-induced nephrotoxicity in rats - role of matrix metalloproteinases 2 and 9. Basic Clin Pharmacol Toxicol. 2009;105(6):416-24. doi: 10.1111/j.1742-7843.2009.00466.x. [PubMed: 19821833].

15. Balakumar P, Rohilla A, Thangathirupathi A. Gentamicininduced nephrotoxicity: Do we have a promising therapeutic approach to blunt it?. Pharmacol Res. 2010;62(3):179-86. doi: 10.1016/j.phrs.2010.04.004. [PubMed: 20434560].

16. Manikandan R, Beulaja M, Thiagarajan R, Priyadarsini A, Saravanan $\mathrm{R}$, Arumugam M. Ameliorative effects of curcumin against renal injuries mediated by inducible nitric oxide synthase and nuclear factor kappa B during gentamicin-induced toxicity in Wistar rats. Eur J Pharmacol. 2011;670(2-3):578-85. doi: 10.1016/j.ejphar.2011.08.037. [PubMed: 21925163]

17. Tavafi M, Ahmadvand $\mathrm{H}$. Effect of rosmarinic acid on inhibition of gentamicin induced nephrotoxicity in rats. Tissue Cell. 2011;43(6):3927. doi: 10.1016/j.tice.2011.09.001. [PubMed: 22000907].

18. Nematbakhsh M, Ashrafi F, Safari T, Talebi A, Nasri H, Mortazavi $\mathrm{M}$, et al. Administration of vitamin $\mathrm{E}$ and losartan as prophylaxes in cisplatin-induced nephrotoxicity model in rats. J Nephrol. 2012;25(3):410-7. doi: 10.5301/jn.5000018. [PubMed: 21928232].

19. Krimer N, Baser KH, Tumen G. Carvacrol rich plants in Turkey. Chem Nat Compounds. 1995;31(1):37-42.

20. Omidi A, Torabi Z, Hassanpoorfard M,Zardast M. Evaluation of protective effect of hydroalcoholic extract of Crocus sativus petals on preventing of gentamicin induced peliosis hepatis and hepatic telangiectasis in rats: Short communication [in Persian]. J Birjand Univ Med Sci. 2013;19(4):455-62.

21. Ruberto G, Baratta MT, Deans SG, Dorman HJ. Antioxidant and antimicrobial activity of Foeniculum vulgare and Crithmum maritimum essential oils. Planta Med. 2000;66(8):687-93. doi: 10.1055/s-2000-9773. [PubMed: 11199122].

22. Alma MH, Mavi A, Yildirim A, Digrak M, Hirata T. Screening chemical composition and in vitro antioxidant and antimicrobial activities of the essential oils from Origanum syriacum L. growing in Turkey. Bio Pharm Bull. 2003;26(12):1725-9. [PubMed: 14646179].

23. Im SJ, Kim JH, Kim MY. Evaluation of bioactive components and antioxidant and anticancer properties of citrus wastes generated during bioethanol production. Nat Prod Commun. 2014;9(4):483-6. [PubMed: 24868862].

24. Zhou K, Li X, Du Q, Li D, Hu M, Yang X, et al. A CAPE analogue as novel antiplatelet agent efficiently inhibits collagen-induced platelet aggregation. Pharmazie. 2014;69(8):615-20. [PubMed: 25158573].
25. Lamarca B. Endothelial dysfunction. An important mediator in the pathophysiology of hypertension during pre-eclampsia. Minerva Ginecol. 2012;64(4):309-20. [PubMed: 22728575].

26. Pedraza-Chaverri J, Gonzalez-Orozco AE, Maldonado PD, Barrera D, Medina-Campos ON, Hernandez-Pando R. Diallyl disulfide ameliorates gentamicin-induced oxidative stress and nephropathy in rats. Eur J Pharmacol. 2003;473(1):71-8. [PubMed:12877940].

27. Safa J, Argani H, Bastani B, Nezami N, Rahimi Ardebili B, Ghorbanihaghjo A, et al. Protective effect of grape seed extract on gentamicininduced acute kidney injury. Iran J Kidney Dis. 2010;4(4):285-91. [PubMed: 20852368].

28. Patel Manali B, Deshpande S, Shah G. Evaluation of efficacy of vitamin $\mathrm{E}$ and N-acetyl cysteine in gentamicin-induced nephrotoxicity in rats. Ren Fail. 2011;33(3):341-7. doi: 10.3109/0886022X.2011.560987. [PubMed: 21401361].

29. Ahmadvand H, Tavafi M, Shahsavari G, Khosrobeigi A, Bagheri S, Abdolahpour F. Hypolipidemic and antiatherogenic effects of Satureja khozestanica essential oil in alloxan-induced type 1 diabetic rats. Zahedan J Res Med Sci. 2013;15(8):26-9.

30. Ahmadvand H, Tavafi M. Amelioration of glomerulosclerosis by Satureja khozestanica essential oil in alloxaninduced diabetic rats. Zahedan J Res Med Sci. 2014;16(10):23-6.

31. Bagheri S, Ahmadvand H, Khosrowbeygi A, Ghazanfari F, Jafari N, Nazem $\mathrm{H}$, et al. Antioxidant properties and inhibitory effects of Satureja khozestanica essential oil on LDL oxidation induced-CuSO(4) in vitro. Asian Pac J Trop Biomed. 2013;3(1):22-7. doi: 10.1016/S22211691(13)60018-0. [PubMed: 23570012].

32. Ahmadvand H, Tavafi M, Khalatbary AR. Hepatoprotective and Hypolipidemic Effects of Satureja Khuzestanica Essential Oil in Alloxaninduced Type 1 Diabetic Rats. Iran J Pharm Res. 2012;11(4):1219-26. [PubMed: 24250556].

33. Abdel-Raheem IT, Abdel-Ghany AA, Mohamed GA. Protective effect of quercetin against gentamicin-induced nephrotoxicity in rats. Biol Pharm Bull. 2009;32(1):61-7. [PubMed:19122282].

34. Zietse R, Zoutendijk R, Hoorn EJ. Fluid, electrolyte and acid-base disorders associated with antibiotic therapy. Nat Rev Nephrol. 2009;5(4):193-202. doi: 10.1038/nrneph.2009.17. [PubMed: 19322184].

35. Gong X, Celsi G, Carlsson K, Norgren S. Protective effects of N-acetylcysteine amide (NACA) on gentamicin-induced apoptosis in LLC-PK1 cells. Ren Fail. 2012;34(4):487-94. doi: 10.3109/0886022X.2012.655684. [PubMed: 22486232].

36. Sahu BD, Tatireddy S, Koneru M, Borkar RM, Kumar JM, Kuncha $M$, et al. Naringin ameliorates gentamicin-induced nephrotoxicity and associated mitochondrial dysfunction, apoptosis and inflammation in rats: possible mechanism of nephroprotection. Toxicol Appl Pharmacol. 2014;277(1):8-20. doi: 10.1016/j.taap.2014.02.022. [PubMed: 24637089]. 